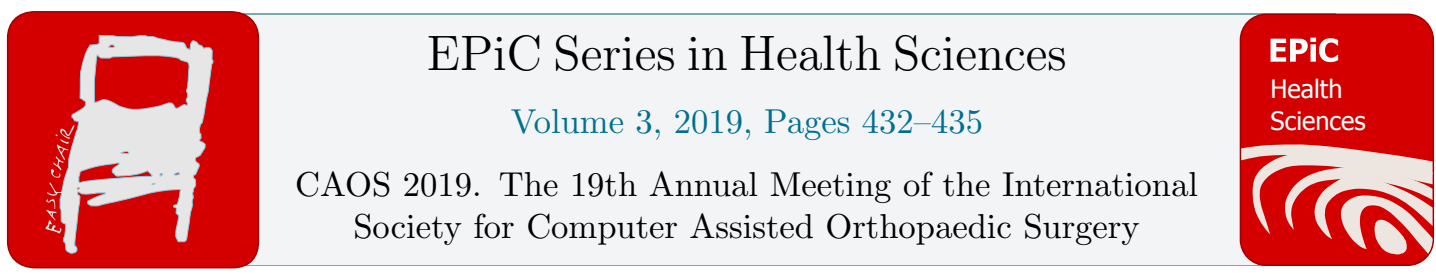

\title{
The Sensitivity of Balancing in Total Knee Surgery
}

\author{
Gabriela Zapata $^{1,2}$, Inigo Sanz-Pena ${ }^{1,3,4}$, Matthias Verstraete ${ }^{5,6}$, Patrick A \\ Meere $^{1}$ and Peter S Walker ${ }^{1,3}$ \\ ${ }^{1}$ Department of Orthopedic Surgery, NYU Langone Orthopedic Hospital, New York, USA \\ ${ }^{2}$ NYU Tandon School of Engineering, Department of Biomedical Engineering, New York, USA \\ ${ }^{3} \mathrm{NYU}$ Tandon School of Engineering, Department of Mechanical and Aerospace Engineering, \\ New York, USA \\ ${ }^{4}$ Universidad de La Rioja, Department of Mechanical Engineering, La Rioja, Spain \\ ${ }^{5}$ Orthosensor Inc., Dania Beach, Florida, USA
}

\section{Introduction}

Since the earliest total knees (TKA) were introduced, limb alignment and soft tissue balancing have been considered as essential factors of the surgical procedure. Experience has shown that inadequate technique in these areas can lead to discomfort, pain, reduced flexion, and instability, which may require revision surgery in extreme cases. Different alignment goals persist, either inserting components perpendicular to the mechanical axis, or an anatomic approach called kinematic alignment (Howell, Papadopoulos, Kuznik, \& Hull, 2013). Balancing itself can be defined as achieving equal collateral ligament tensions with the same values at all flexion angles, or more recently and in concert with kinematic alignment, maintaining higher medial than lateral forces, the lateral forces diminishing with flexion angle. Various methods have been used by surgeons to achieve the balancing goals, including spacer blocks, tensors, and recently, instrumented tibial trials, but there are no current techniques for measuring ligament forces (D'Lima \& Colwell, 2017). The more quantitative methods of balancing have facilitated a systematic approach, which can produce improved outcomes (Meere, Schneider, \& Walker, 2016; Verstraete, Meere, Salvadore, Victor, \& Walker, 2017). Nevertheless, accurate balancing has been difficult to achieve due to the high sensitivity of the forces to even small changes in the ligament lengths (Walker, Meere, \& Bell, 2014). Such changes can occur due to component shapes or the angles and levels of the bone cuts. Hence the first goal of this study was to focus on the effects of errors of component placement and on different surgical alignment techniques, kinematic and mechanical, on the balancing. The second goal was to study the relationship between the collateral ligament forces, the distraction forces, and the condylar contact forces, to determine if there was equivalence between the different balancing methods. 


\section{Methods}

A test rig (Figure 1) was designed to measure the changes in condylar contact forces of a standard TKA resulting from errors in femoral component placement, as well as differences in alignment techniques. With the components in place, a motor was used to flex and extend the knee, while the condylar forces were measured using an instrumented tibial component, and the ligament forces were measured with transducers. The test rig modeled the collateral ligaments using a unique method where they were extended out of the joint, such that their stiffness and pre-tension forces could be readily adjusted and measured. The LCL was a single cable, but the MCL was modelled by anterior and posterior fibers. Ideal femoral component placement was defined as the sagittal contours of the femoral component exactly matching the anatomic condyles. Simulated placement errors relative to the anatomic condyles were proximal (excessive distal femoral resections), distal, anterior, and posterior. These positions were modelled by $2 \mathrm{~mm}$ movements of the ligament attachment points on the femur relative to the center of rotation. The condylar force values for ideal anatomic placement were obtained as a reference, while the condylar force values for the different femoral placements were measured relative to the ideal. To compare the different balancing measurement methods, regression analysis was used to study the correlation between the ligament and condylar forces, while force analysis was used to compare the distraction forces.

\section{Results}

Condylar contact forces were significantly influenced by $2 \mathrm{~mm}$ errors in femoral component placement. For a distal placement error (too little cut from the distal femur), there were only small changes in the contact forces except for a decrease in the lateral force in higher flexion. On the medial side, anterior and proximal errors decreased the compartmental loads to zero while a posterior error increased the force almost $50 \%$. On the lateral side, proximal and posterior errors significantly increased the forces by $+450 \%$ and $+470 \%$, respectively. However, anterior error decreased the compartmental loads. For the surgical technique, mechanical alignment decreased the lateral compartmental forces to zero while medial forces were similar for all alignment variations. The combined medial and lateral ligament and contact forces showed a linear relation with a correlation coefficient of 0.98 . For the medial and lateral sides separately the correlations were 0.85 and 0.88 respectively, with more than $80 \%$ of points within a $\pm 25 \%$ deviation from the linear relations. This deviation from the linear correlation is linked to differences in medial-lateral femoral-tibial contact point locations at different flexion angles.

\section{Conclusions}

The ideal situation was kinematic alignment (Howell, Papadopoulos, Kuznik, \& Hull, 2013), which could reproduce the condylar contact forces seen in intact anatomic knees (Verstraete, Meere, Salvadore, Victor, \& Walker, 2017). Relative to that ideal, the least condylar force errors occurred when the femoral component was placed too distally, for the kinematic alignment bone cuts. For mechanical alignment, distal bone resections needed to be $10 \mathrm{~mm}$ or more to maintain adequate load levels through the range of motion. Within balancing accuracies generally achieved at surgery, the collateral ligament forces were linearly correlated to the condylar contact forces. These forces can also be equally correlated to the distraction forces as well as the moments at which condylar lift-off would occur from 
varus-valgus moments. This indicated a unification of the different balancing parameters, and hence such quantitative methods can be used interchangeably.

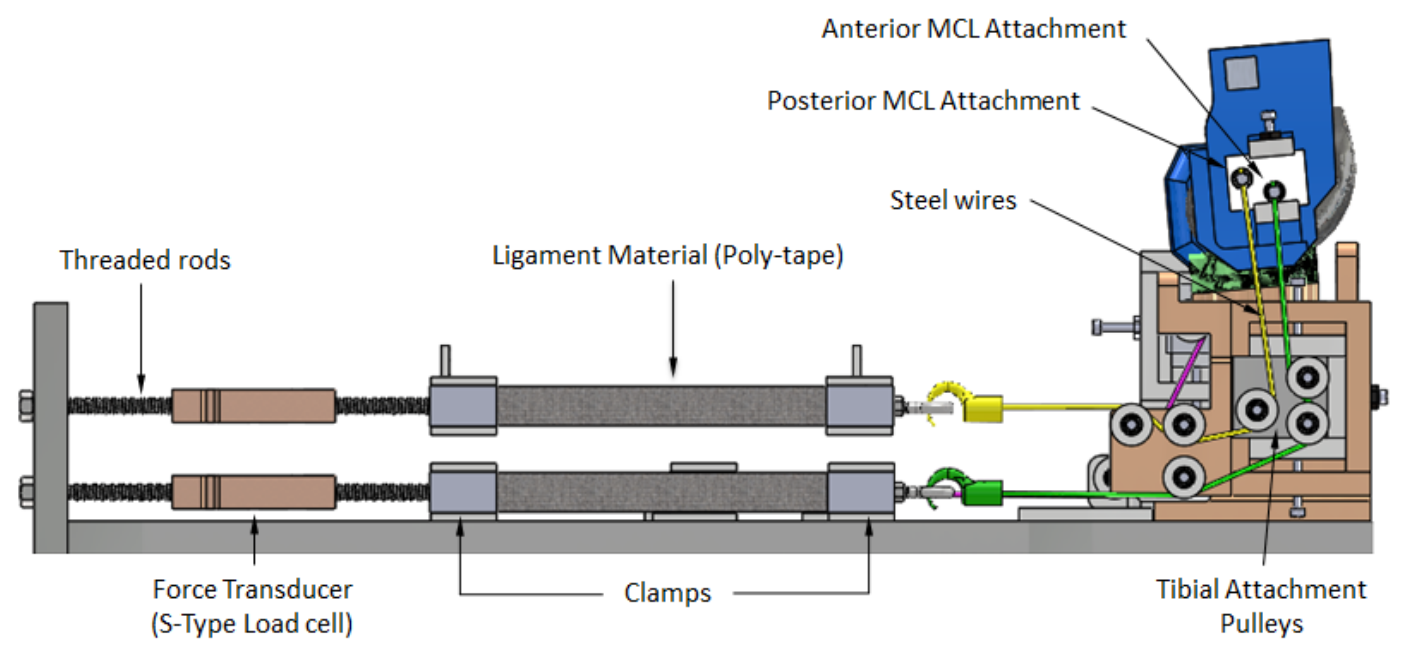

Figure 1. Side view of the rig used to model the total knee and obtain the lateral and medial ligament and condylar forces.

\section{References}

D'Lima, D., \& Colwell, C. (2017). Intraoperative Measurements and Tools to Assess Stability. Journal of the American Academy of Orthopaedic Surgeons, S29-S32.

Howell, S., Papadopoulos, S., Kuznik, K., \& Hull, M. (2013). Accurate alignment and high fuction after kinematically aligned TKA. Knee Surg Sports Traumat Arthrosc, 2271-2280.

Meere, P., Schneider, S., \& Walker, P. (2016). Accuracy of Balancing at Total Knee Surgery Using Instrumented Tibial Trial. Journal of Arthroplasty, 1938-1942.

Verstraete, M., Meere, P., Salvadore, G., Victor, J., \& Walker, P. (2017). Contact forces in the tibiofemoral joint from soft tissue tensions: Implications to soft tissue balancing in total knee arthroplasty. Journal of Biomechanics, 195-202.

Walker, P., Meere, P., \& Bell, C. (2014). Effects of surgical variables in balancing of total knee replacments using an instrumented tibial trial. Knee, 156-161.

\section{Acknowledgements \& Disclosures}

The research was funded from an Educational Grant from Orthosensor Inc., Dania Beach, FL through NYU Langone Medical Center. PA Meere and M Verstraete have commercial interests in 
Orthosensor Inc. PS Walker receives royalties from Zimmer and Stryker for total knee design. G Zapata and I Sanz-Pena have no disclosures. 